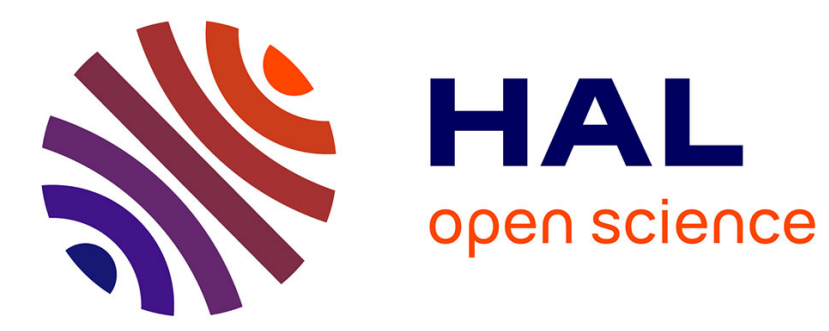

\title{
A Portable Interface for Tangible Exploration of Volumetric Data
}

\author{
Paul Issartel, Florimond Guéniat, Mehdi Ammi
}

\section{To cite this version:}

Paul Issartel, Florimond Guéniat, Mehdi Ammi. A Portable Interface for Tangible Exploration of Volumetric Data. ACM 20th Symposium on Virtual Reality Software and Technology (VRST), Nov 2014, Edinburgh, United Kingdom. pp.209-210, 10.1145/2671015.2671130 . hal-02505400

\section{HAL Id: hal-02505400 \\ https://hal.science/hal-02505400}

Submitted on 11 Mar 2020

HAL is a multi-disciplinary open access archive for the deposit and dissemination of scientific research documents, whether they are published or not. The documents may come from teaching and research institutions in France or abroad, or from public or private research centers.
L'archive ouverte pluridisciplinaire HAL, est destinée au dépôt et à la diffusion de documents scientifiques de niveau recherche, publiés ou non, émanant des établissements d'enseignement et de recherche français ou étrangers, des laboratoires publics ou privés. 


\section{A Portable Interface for Tangible Exploration of Volumetric Data}

\author{
Paul Issartel* \\ LIMSI-CNRS \\ Université Paris Sud
}

\author{
Florimond Guéniat ${ }^{\dagger}$ \\ LIMSI-CNRS \\ Université Paris Sud
}

\author{
Mehdi Ammi ${ }^{\ddagger}$ \\ LIMSI-CNRS \\ Université Paris Sud
}

\begin{abstract}
Exploration of volumetric data is an essential task in many scientific fields. However, the use of standard devices, such as the 2D mouse, leads to suboptimal interaction mappings. Several VR systems provide better interaction capabilities, but they remain dedicated and expensive solutions. In this work, we propose an interface that combines tangible tools and a handheld device. This configuration allows natural and full 6 DOF interaction in a convenient, fully portable and affordable system. This paper presents our design choices for this interface and associated tangible exploration techniques.
\end{abstract}

\section{Introduction}

Exploration of complex volumetric datasets is of crucial importance in many scientific fields. Currently, most software for visualizing volumetric data are designed for desktop computers. They are thus constrained by the limitations of mouse interaction. The mouse only provides two degrees of freedom (DOF), while many exploration tools require up to $6 \mathrm{DOF}$ ( 6 for 3D navigation, 3 for pointing, etc.). Therefore, the interaction has to be decomposed in smaller steps which is suboptimal and increases complexity.

Other approaches are based on virtual reality [Qi et al. 2006; Laha et al. 2014]. In such interfaces, the data is in the same 3D space as the user. This lets the user directly interact with the data in 6 DOF, overcoming the limitations of the desktop mouse. However, these interfaces also require dedicated and complex hardware (CAVE, head-mounted displays, trackers...). Because of that, they remain impractical and are too often dismissed by intended users for everyday work.

In this work, we present an interface that addresses the above issues. For the practicality aspect, we base our approach on a readily available and fully portable device: a tactile tablet. To support direct and natural 6 DOF interaction, we propose to combine the tactile tablet with tangible objects. These simple real-world props are directly coupled with the data volume and with exploration tools, allowing the user to interact with the dataset as if it were a real object.

\section{Hardware and software platform}

A key point of our approach is to use common, off-the-shelf hardware. The interface was implemented on an Android tablet: a Toshiba AT270. It has a 7.7" screen and weighs $332 \mathrm{~g}$. This form

\footnotetext{
*paul.issartel@limsi.fr

†contact@gueniat.fr

${ }^{\ddagger}$ mehdi.ammi@limsi.fr
}

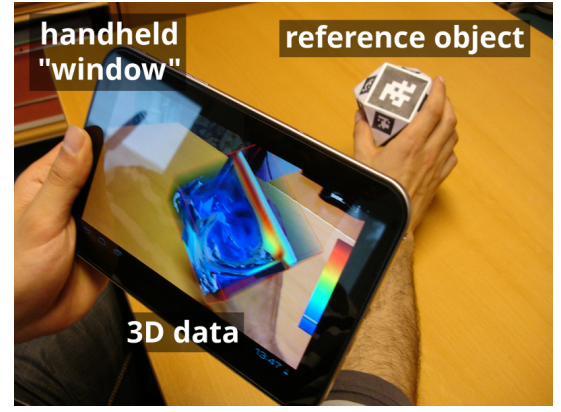

Figure 1: Visualizing and manipulating a volume behind the tablet, turned into a handheld see-through window.

factor was chosen to be easy to hold in one hand when necessary, while still having a relatively large screen for comfortable viewing.

We turn the tablet into a handheld see-through window by capturing the background environment from the rear camera and displaying it on the screen. Seeing the background environment is particularly helpful to manipulate tangible objects relative to each other. The video stream is also analyzed in real-time to locate tangible objects, using ARToolKit ${ }^{1}$ markers. Each tangible object is equipped with multiple such markers. This enables tracking to be maintained regardless of its orientation, since objects are meant to be freely manipulated by the user. When a tangible object is detected, the corresponding virtual object (dataset or exploration tool) is displayed on the screen at the same position and orientation.

\section{Tangible objects}

We designed two tangible objects for this interface: the reference object and the stylus. The tablet itself can be considered as a third tangible object.

The reference object represents the dataset. It acts as a physical handle for the virtual volume. When this object is observed through the handheld window, the data volume is superimposed on it (Figure 1). To begin exploration, the reference object simply needs to be brought behind the tablet - or, equivalently, the tablet has to be pointed toward this object. Manipulating the reference object lets users manipulate the data volume directly and naturally, with bare hands, as if it were a real-world object.

The stylus (visible in Figure 2(b)) is a multipurpose exploration device. It can be associated with different virtual tools. All these tools have in common the need to select a precise location in the volume. The elongated shape of the stylus "suggests" this usage: the handle makes it look like a pen, and its tip is an effector. Hence the stylus benefits from the dexterity previously acquired from the use of pens. A few controlled movements are enough to reach quickly and accurately any point in the volume. One issue inherent to tangible objects is that the stylus and the reference object cannot interpenetrate. This would prevent the stylus tip from reaching some locations in the data. To overcome this problem, the virtual tip (effector) is shifted forward relative to the physical tip.

\footnotetext{
${ }^{1}$ http://www.hitl.washington.edu/artoolkit/
} 


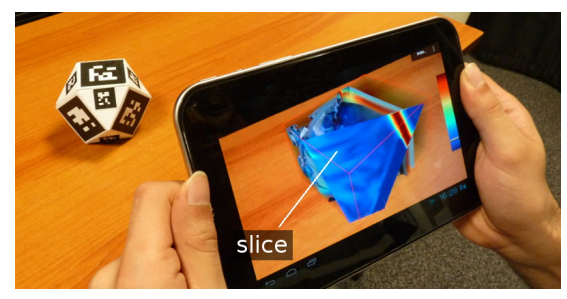

(a) Tablet-based slicing

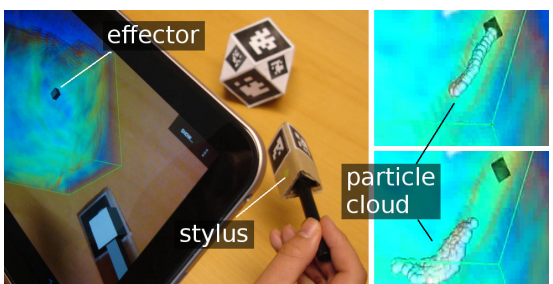

(b) Particle tracing

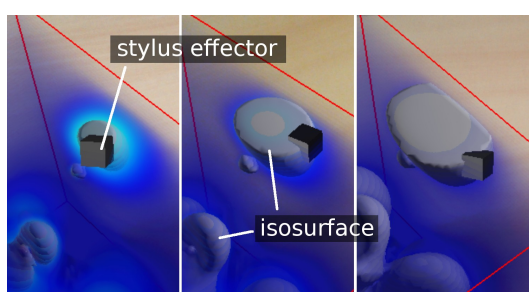

(c) Isosurface picking

Figure 2: Illustration of some of the interaction techniques supported by our system.

\section{Interaction techniques}

\subsection{Manipulating the dataset}

Interactively exploring a volume generally requires to observe it from different angles. In our interface, rotating the dataset can be achieved by rotating the reference object, directly with the hand. Visual feedback is provided by the tablet (Figure 1). Since volume datasets are generally cuboids, we initially assumed a cube would be the best shape for the reference object. Each face corresponds to a quarter turn or half turn, which provides meaningful resting positions for the object. However, under some orientations, there would be only one face visible from the camera. And this face could be obscured by fingers, hiding the last visible marker. We thus altered the shape to increase the odds that at least one marker would remain visible during manipulation, while retaining the original "quarter turn" faces. This new shape (visible in Figure 2(a)) is a cuboctahedron.

\subsection{Volume slicing}

Slicing a volume along a plane is a technique commonly used to visualize structures within the dataset. The combination of a tablet and tangible objects brings new possibilities for creating and manipulating slice planes. The two slicing techniques described here were first presented and described in more detail in [Issartel et al. 2014].

The first metaphor is to use the tablet itself as a tangible object. It has a planar shape, and its position and orientation relative to the data are tracked. Thus the tablet is actually a tangible plane directly controlled by the user in the 3D space. This tangible plane defines a slice through the data volume. A slice plane can be obtained by positioning the tablet and the reference object relative to each other. The result of slicing is visible in real-time through the handheld window (Figure 2(a)).

The second slicing mode uses the stylus as a slicing tool. In this metaphor, a virtual "blade" is attached to the stylus. The blade defines a slice through the data volume. As in the previous mode, the slice is updated in real-time, giving the impression of effectively slicing the virtual data with a physical tool.

\subsection{Isosurface picking}

When working with scalar fields, isosurfaces can be used to identify and isolate areas of interest. An isosurface is a surface built from all points that have a given value. Most visualization software lets users manually enter the desired value for the isosurface. However this value is not necessarily known during exploration. In such case, it may be more appropriate to generate the isosurface according to the visible structures in the data volume.
In our interface, the stylus can be used to directly point to a 3D location within the volume. Then, an isosurface is computed from the scalar value at this point (Figure 2(c)). Additionally, we provide an option to continuously display a low-resolution preview of the isosurface in real-time, during displacement of the stylus, until the desired value is reached. Thus, this technique allows users to interactively explore a range of isovalues.

\subsection{Particle tracing}

Some volume datasets are actually vector fields. Particle tracing is a technique to interactively explore these datasets. It consists of generating a number of particles at a given location within the volume. Each particle then follows the vector field from this starting point. Particles are generated with a slight temporal or spatial offset, so their trajectories do not completely overlap. The moving particle cloud provides a way to visually identify structures in the vector field. A similar technique, known as "seeding", is also used to explore real (physical) fluid flows, with real particles such as smoke.

This technique provides another opportunity to demonstrate the advantages of our interface concept. In our interface, the stylus becomes a particle source. The stylus effector defines the location where particles are generated, and can be positioned within the dataset by looking through the handheld window (Figure 2(b)). For an expert, using the stylus is just like using a real seeding device, except that it "seeds" a virtual flow.

\section{Conclusion}

This paper presents an interface for the exploration of volumetric datasets, designed to support direct and natural 3D interaction with the data, in a convenient way. Convenience is attained by using fully portable and widespread hardware - a tactile tablet - as a handheld augmented reality window. Direct and natural 3D interaction is achieved by manipulating simple tangible objects behind the handheld tablet. Our work opens new perspectives to make interactive exploration of volumetric data more accessible to end users.

Acknowledgements: this work was supported by RPE.

\section{References}

IsSARTEl, P., GuÉNiAT, F., AND AMMI, M. 2014. Slicing techniques for handheld augmented reality. In Proc. IEEE 9th Symposium on $3 D$ User Interfaces (3DUI), IEEE, 39-42.

LAHA, B., Bowman, D., AND Socha, J. 2014. Effects of VR system fidelity on analyzing isosurface visualization of volume datasets. IEEE TVCG 20, 4 (Apr.), 513-522.

Qi, W., Taylor II, R. M., Healey, C. G., and Martens, J.B. 2006. A comparison of immersive HMD, fish tank VR and fish tank with haptics displays for volume visualization. In Proc. $A P G V$, ACM, 51-58. 